\title{
Public Health Applications Research Branch
}

National Cancer Institute

\section{Source}

National Cancer Institute. Public Health Applications Research Branch. NCI Thesaurus. Code C19281.

Part of the Division of Cancer Prevention and Control. develop a research and applications program to support public health agencies in their cancer control efforts. regularly and systematically assess community health needs, develop comprehensive public health policies based on the use of scientific knowledge, and assure the provision of services necessary to meet agreed-upon goals. 